\title{
Resection of a Huge Fourth-Recurrence Retroperitoneal Liposarcoma
}

\author{
Yong Zhou ${ }^{\mathrm{a}}$, Fu Yu Li ${ }^{\mathrm{a}}$, Ri Hua Xua $\mathrm{Xu}^{\mathrm{a}}$, Yong Qiong Zhanga, \\ Hong Liu ${ }^{\mathrm{a}}$, Ting Jiang ${ }^{\mathrm{a}}$, Wen $\mathrm{Li}^{\mathrm{a}}$
}

\begin{abstract}
Resection of retroperitoneal liposarcomas continues to be a challenge for the surgeon, in particular given their large size and involvement of vital organs. We present our experience about the resection of huge fourth-recurrence retroperitoneal liposarcomas, this dumbbell-shaped mass occupied portovena-caval gap, encapsulated the portal vein and was closed attached to inferior vena cava. It was almost impossible to push the whole mass to any side for en block resection because both left and right part of this mass all reached $20 \mathrm{~cm}$. So we decided to firstly resect the right part of dumbbell-shaped mass, dissect the link part from behind the portal vein, followed by pushing it to the left side and then resect together with the left part. This method made the complete resection easier and safer, of which avoid the occurrence of uncontrollable massive hemorrhage.
\end{abstract}

Keywords: Retroperitoneal liposarcomas; Surgery; Recurrence

\section{Introduction}

Liposarcomas are rare cancers that account for approximately $15 \%$ of adult soft-tissue tumors. It should be specially stressed here that liposarcoma originate from the mesenchymal tissue and not from the fatty tissue. Retroperitoneal liposarcoma is the most common case that represents $40 \%$ of all soft tissue sarcomas occur in the retroperitoneum $[1,2]$.The

\footnotetext{
Manuscript accepted for publication March 23, 2012

${ }^{\mathrm{a}}$ Department of Hepatobiliary Surgery, West China Hospital of Sichuan University, Chengdu, China

${ }^{\mathrm{b}}$ Corresponding author: Fu Yu Li, Department of Hepatobiliary Surgery, West China Hospital of Sichuan University, Chengdu 610041, Sichuan Province, China. Email: 1fy_74@vip.163.com
}

doi:10.4021/jmc630e retroperitoneal space contains, embedded in a meshwork of loose connective tissue, the adrenal glands, kidneys, and ureters; the aorta and its branches; the inferior vena cava and its tributaries; and numerous lymph nodes. This potentially large space allows the tumor to grow silently before clinical signs and symptoms appear. It has been reported that $20 \%$ of the tumors are $>10 \mathrm{~cm}$ at the time of diagnosis. Due to the large retroperitoneal space, patients with retroperitoneal liposarcoma have no obvious symptoms in the early stages until the mass develops enough to press or invade the neighboring structures. This late-diagnosis character therefore reduces the rate of complete resection. Even worse, RI has a high local recurrence rates compared with the liposarcomas occur in other part $[3,4]$. According to a 72 cases report from Neuhaus, local recurrence rate could be as high as 65\% [5], while the recurrence rate for differentiated RI in the Singer's report even reached $83 \%$ [6]. Our recent experience with one case of fourth recurrence RI has prompted us to report the surgical management of this huge dumbbell-shaped tumor that occupied portovena gap, encapsulated the portal vein and was closed attached to inferior vena cava.

\section{Case Report}

From May 2000 and April 2009, this 40-year-old woman has surveyed four-times tumor resections including her left kidney due to the recurrence of retroperitoneal liposarcoma in the outer court. After this admission, her MRI showed that a huge middle-upper retroperitoneal mass occupied hepatorenal recess, hepatogastric ligament, hepatoduodenal ligament, the gap between the portal vein and the inferior vena cava; the mass's upper pole reached the first portal hepatis; right bottom reached the fourth lumbar flanges, oppressed and displaced the portal vein and inferior vena cava and mesenteric root. Intraoperative foundation showed that this dumbbellshaped mass had the integrated encapsulate in its most part, except that portion of mass had no obvious coating for the existence of scar and dense adhesion with the surrounding tissue due to prior operations (Fig. 1). The right upper part of this dumbbell-shaped mass was about $20 \mathrm{~cm} \times 15 \mathrm{~cm}$ in size, encapsulated the portal vein and was densely attached 


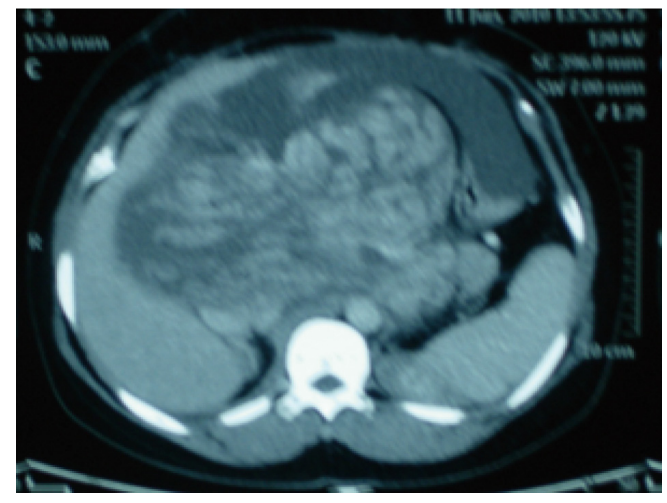

Figure 1. Her MRI revealed a dumbbell-shaped retroperitoneal liposarcomas occupied the gap between the portal vein and the inferior vena cava, encapsulated the portal vein and densely adherent to inferior vena cava.

to inferior vena cava. The left part of this mass located in hepatogastric ligament and was about $20 \mathrm{~cm} \times 10 \mathrm{~cm}$ in size. Unfortunately, the link part of this dumbbell mass occupied the whole gap between the portal vein and inferior vena cava. Except for this, there are $8 \mathrm{~cm}$ part of this mass that was closely adhered to portal vein and inferior vena cava.

For these reasons, the en block resection seem extremely difficulty, there would be a greater probability of uncontrollable massive hemorrhage if we perform an en block resection of this dumbbell-shaped tumor by force. Thus we decided to firstly resect its right part and found that the right-side attachment with inferior vena cava was only the close adhesion instead of the involvement. After careful dissection, the right part of mass was removed from right-side of portal vein and inferior vena cava (Fig. 2). Then we now have good vision and turn to the careful dissection of the link part of this dumbbell mass from behind portal vein. Subsequently, the left part of mass was fully mobilized from left lobe of liver and gastric lesser curvature. After finishing these steps, the link part located behind portal vein could be whole pushed to left side of hepatoduodenal ligament and thus enable the successful enblock resection together with the left part. Finally, the surrounding adipose and tissue were thoroughly cleared. Intraoperative bleeding was about $1500 \mathrm{~mL}$, and her postoperative recovery was smooth. Postoperative pathologic diagnosis was liposarcoma accompanied by dedifferentiate and S - $100(+)$, CD34 (+), CD117 (-).

\section{Discussion}

Liposarcoma belongs to a kind of soft tissue sarcomas, accounting for malignant sarcomas $1 \%$. Most of retroperitoneal liposarcoma (RI) have a complete coating and show the dilatability growth mode and low invasive capability; hence most of them can be complete resection. So far, surgery is still the only way to that RI, which emphasizes the complete surgical

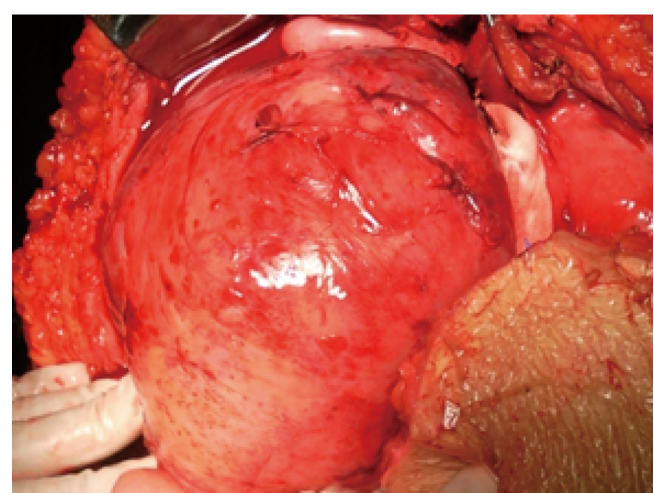

Figure 2. After careful dissection, the right part of this dumbbell-shaped was freed from the adjacent viscera and inferior vena cava.

resection $[7,8]$. The thoroughness and integrity of first excision is pivotal factor to determine its curative effect. Most of literatures agreed that the surrounding fat tissue should be thoroughly cleared, because any traces of surrounding adipose tissue are likely to conceal the variably-sized satellite focal or tumor cell. Additionally, the encapsulate of RI is actually a pseudocapsule formed by the surrounding connective tissue and tumor cells, so tumor cells often invade the pseudocapsule or surrounding tissue and even led to appearance of the skip spread. For these reasons, RI excision should include the surrounding normal tissues and involved organ $[9,10]$. Due to the quick growth of tumor, neighboring large blood vessels and viscera were prone to be oppressed or even invaded. Once the invasion happens, the combined resection of multiorgan or part of the viscera is recommended on the condition of patients' tolerance, so as to achieve the radical resection $[11,12]$. According to 40 radical resections of RI from Serio's report, $62 \%$ patients ( 25 cases) had to surfer the combined resection of the surrounding viscera, while major vascular resection had to be performed in another 17 cases [13]. Complete resection can not only enhance the patient's survival time, but also reduce tumor recurrence rates. Relative studies indicated that the 5-year survival rate of complete excision and non-complete excision was respectively $75 \%$ and $34 \%$, of which emphasizes the importance the radical resection $[6,14]$.

The specialty of this case lies in: this patient needed a reoperation for the fifth recurrence, part of mass had no obvious capsule for the prior operations' scar and dense adhesion, a consequence of which increased the resection difficulty; more specifically, the link part of this dumbbell mass occupied the whole gap between the portal vein and the inferior vena cava, and encapsulated the portal vein; except for this, there was $8 \mathrm{~cm}$ part of this mass that was closely attached to portal vein and inferior vena cava; the diameter of both left and right part of this mass all reached $20 \mathrm{~cm}$. For these reasons, it is almost impossible to push the whole mass to any side for en block resection. If we perform an en block resec- 
tion of this dumbbell-shaped tumor by force, there would be a greater probability of uncontrollable massive hemorrhage. For this kind of dumbbell RI adjacent to major vessels, we should consider firstly resecting part of mass, pushing the rest part to the other side and then resecting together, of which make the complete resection more easy and safe. Finally, the clear of surrounding adipose and tissue must be performed so as to ensure maximum degree of the thoroughness and integrity excision, and to avoid the post-operation relapse $[5,9,14]$.

The Current difficulty of RI treatment is high postoperative local-recurrence rate. The characteristic of RI recurrence is in situ and multiple recurrences, while distant metastases seldom occur $[15,16]$. Some studies even believed that local recurrence rate of RI were still high after radical resection. According to the 72 cases report from Neuhaus, the 3-year recurrence rate after complete resection still reached $65.3 \%$. Wang's report agreed this point with a $65 \%$ of 3 -year recurrence rate. Singer's study indicated the positive margin and organization subtypes (especially for dedifferentiate type) were closely related to local recurrence, and were important prognostic factors affecting survival; the 3-year local recurrence rate of dedifferentiate liposarcoma as in this case could be as high as $83 \%[5-8,11,17]$. At present, most of scholars think the reason of recurrence lies in the following four respects: (1) the large size tumor often invade surrounding viscera or encapsulate the major vessels, which bring difficult to obtain a negative pathology margin even with the multiorgan or vascular resection; (2) Of note, RI actually do not have a real capsule. The expansile growth mode of RI can push surrounding normal tissues to form "pseudocapsule", which make the complete resection more difficult; (3) the infiltrated growth of worse pathology subtypes also bring difficulty to the thorough removed; (4) there are many sizes of "satellite lesions" hiding around primary tumors, and these satellite lesions cannot be easily recognized under naked eyes, and are easy to be slip through net, leading to the unthrough resection $[2,3,18,19]$. For these reasons, some scholars like Zhang et al even considered that the recurrence are impossible to be avoided even using the multiorgan resection in order to meet with the resection scope [20]. Our personal experience agreed that those recurrent cases should better be earlier detected and resected, even the palliative resection as "dissect within the capsule", also can help reduce the tumor burden and prolong survival time [21]. For those unresectable tumors, Serio et al [13] advocated the first using of chemotherapy or radiation therapy followed by surgery, so as to strive for the chance of complete or partial resection and thus improving survival. The author thinks that, although RI is characterized by high local recurrence, we should not easily give up the chance of reoperation because the pathology of those multiple recurrence cases is usually well differentiation type. Supportive of this idea came from recent literatures indicated that survival time of fourth or more resection for this kind of well-differentiation liposarcoma had no obvious difference with that of second resection $[2-6,20]$. As for the using of chemoradiation, it remains controversial. Stoeckle et al believed postoperative radiotherapy could reduce local recurrence rate, the combination of surgery and radiation therapy should be the most satisfactory choice. However, some scholars disagreed with this point and suspected the actual effectiveness of chemoradiation on recurrent RI till now $[18,21]$.

In brief, RI overall survival depends on the improvement of early detection and surgical resection which emphasize the importance of radical resection. On the primes of no postoperative complications, the surrounding fat and normal tissues should be cleaned as through as possible. Relapse is pivotal factor to affect its curative effect, however, the actively treatment should be adapted to those recurrent and metastatic RI so as to prolong survival time [5-8, 11-14, 22].

\section{Conflict of Interest}

All authors declare that they have no conflict of interest.

\section{References}

1. Kim ES, Jang SH, Park HC, Jung EH, Moon GB. Dedifferentiated liposarcoma of the retroperitoneum. Cancer Res Treat. 2010;42(1):57-60.

2. Park JO, Qin LX, Prete FP, Antonescu C, Brennan MF, Singer S. Predicting outcome by growth rate of locally recurrent retroperitoneal liposarcoma: the one centimeter per month rule. Ann Surg. 2009;250(6):977-982.

3. Mussi C, Collini P, Miceli R, Barisella M, Mariani L, Fiore M, Casali PG, et al. The prognostic impact of dedifferentiation in retroperitoneal liposarcoma: a series of surgically treated patients at a single institution. Cancer. 2008;113(7):1657-1665.

4. Nathan H, Raut CP, Thornton K, Herman JM, Ahuja N, Schulick RD, Choti MA, et al. Predictors of survival after resection of retroperitoneal sarcoma: a populationbased analysis and critical appraisal of the AJCC staging system. Ann Surg. 2009;250(6):970-976.

5. Neuhaus SJ, Barry P, Clark MA, Hayes AJ, Fisher C, Thomas JM. Surgical management of primary and recurrent retroperitoneal liposarcoma. $\mathrm{Br} \mathrm{J}$ Surg. 2005;92(2):246-252.

6. Singer S, Antonescu CR, Riedel E, Brennan MF. Histologic subtype and margin of resection predict pattern of recurrence and survival for retroperitoneal liposarcoma. Ann Surg. 2003;238(3):358-370; discussion 370-351.

7. Santos CE, Correia MM, Thuler LC, Rosa BR, Accetta A, de Almeida Dias J, de Mello EL. Compartment surgery in treatment strategies for retroperitoneal 
sarcomas: a single-center experience. World J Surg. 2010;34(11):2773-2781.

8. Chen CQ, Yin L, Peng CH, Cai Y, Li YF, Zhao R, Zhou HJ, et al. Prognostic factors of retroperitoneal soft tissue sarcomas: analysis of 132 cases. Chin Med J (Engl). 2007;120(12):1047-1050.

9. Sato T, Yamaguchi T, Azekura K, Ueno M, Ohyama $\mathrm{S}$, Ohya M, Yamamoto J, et al. Repeated resection for intra-abdominal and retroperitoneal liposarcomas: longterm experience in a single cancer center in Japan. Int Surg. 2006;91(5):267-271.

10. Dalal KM, Kattan MW, Antonescu CR, Brennan MF, Singer S. Subtype specific prognostic nomogram for patients with primary liposarcoma of the retroperitoneum, extremity, or trunk. Ann Surg. 2006;244(3):381-391.

11. Fu Q. Huge retroperitoneal liposarcoma: a case report. Chin Med J (Engl). 2007;120(12):1117-1118.

12. Gronchi A, Lo Vullo S, Fiore M, Mussi C, Stacchiotti S, Collini P, Lozza L, et al. Aggressive surgical policies in a retrospectively reviewed single-institution case series of retroperitoneal soft tissue sarcoma patients. J Clin Oncol. 2009;27(1):24-30.

13. Serio G, Tenchini P, Nifosi F, Iacono C. Surgical strategy in primary retroperitoneal tumours. $\mathrm{Br} \mathrm{J}$ Surg. 1989;76(4):385-389.

14. Doglietto GB, Tortorelli AP, Papa V, Rosa F, Bossola M, Prete FP, Covino M, et al. Giant retroperitoneal sarcomas: a single institution experience. World J Surg. 2007;31(5):1047-1054.

15. Han HH, Choi KH, Kim DS, Jeong WJ, Yang SC, Jang
SJ, Choi JJ, et al. Retroperitoneal giant liposarcoma. Korean J Urol. 2010;51(8):579-582.

16. Goertz RS, Lenfers BH, Goertz GH. Huge liposarcoma of the left retroperitoneum. Am J Surg. 2009;197(6):e5960.

17. Sun YM, Wang CF, Tian YT, Zhao DB, Shan Y, Zhao P. [Surgical treatment and prognosis of primary retroperitoneal malignant tumor]. Zhonghua Yi Xue Za Zhi. 2009;89(38):2699-2701.

18. Stoeckle E, Coindre JM, Bonvalot S, Kantor G, Terrier P, Bonichon F, Nguyen Bui B. Prognostic factors in retroperitoneal sarcoma: a multivariate analysis of a series of 165 patients of the French Cancer Center Federation Sarcoma Group. Cancer. 2001;92(2):359-368.

19. Alldinger I, Yang Q, Pilarsky C, Saeger HD, Knoefel WT, Peiper M. Retroperitoneal soft tissue sarcomas: prognosis and treatment of primary and recurrent disease in 117 patients. Anticancer Res. 2006;26(2B):15771581.

20. Zhang C. [The patho-morphology and biological behavior of repeatedly recurrent liposarcoma in retroperitoneum]. Zhonghua Bing Li Xue Za Zhi. 1999;28(1):12-15.

21. Thomas DM, O'Sullivan B, Gronchi A. Current concepts and future perspectives in retroperitoneal soft-tissue sarcoma management. Expert Rev Anticancer Ther. 2009;9(8):1145-1157.

22. Hosaka A, Masaki Y, Yamasaki K, Aoki F, Sugizaki $\mathrm{K}$, Ito E. Retroperitoneal mixed-type liposarcoma showing features of four different subtypes. Am Surg. 2008;74(12):1202-1205. 